\title{
Ejaculation obtained by intracavernous papaverine in a cervical spinal cord injury patient resulting in pregnancy and childbirth. Case report
}

\author{
T Otani MD, S Kai MD, M Narushima MD \\ Department of Urology, Chubu-Rosai Hospital, 1-10-6, Koumei, Minato, Nagoya, Japan.
}

\begin{abstract}
A 28 year old male sustained a cervical spinal cord injury (C5, complete) from a traffic accident and was impotent. He started intracavernous injection therapy, by his partner, of papaverine hydrochloride for impotence, restoring ejaculation. His partner had a successful pregnancy after normal sexual intercourse, and delivered a mature female child weighing $3330 \mathrm{~g}$. To our knowledge this is a rare case of a patient with a complete spinal cord injury fathering a child by the above technique.
\end{abstract}

Keywords: spinal cord injury; sexual dysfunction; impotence; papaverine hydrochloride; successful pregnancy.

\section{Introduction}

Intracavernous injection of a vasoactive drug is widely used not only for the diagnosis of impotence but also for its treatment. ${ }^{1}$ But ejaculation can hardly be expected from this therapy when used for the treatment of organic impotence. It is rare to read of a documented case of this therapy successfully leading to pregnancy and childbirth. Such a patient is described in this paper.

\section{Case presentation}

A 28 year old man with a cohabitant (nurse) was involved in February 1985 in a traffic accident and sustained an injury at C5 level resulting in quadriplegia. Sensory function was partially retained in the upper limbs. There was incomplete paralysis of the upper limbs. The bulbocavernous reflex and anal tone were weak and there were spasms of the legs. He had an indwelling catheter for 5 months, followed by intermittent catheterization for the following 5 months, and then managed self catheterization twice daily and voiding by the Valsalva technique. Cystometry showed an overactive bladder; uninhibited detrusor contraction appeared at $100 \mathrm{ml}$. In January 1987 he underwent transurethral cystolithotripsy. In June 1990 he was operated on for a right ureteral stone. After the injury the patient retained reflex erection, but erotic erection and ejaculation were absent. Coitus was impossible.

He desired to have a child and on 19 September 1988 electroejaculation was attempted with a ring-like dipole electrode, ${ }^{2}$ and 7 minutes later erection occurred with an accompanying $0.3 \mathrm{ml}$ ejaculation (spermatozoa: $0 / \mathrm{ml}$ ). Unfortunately he also experienced severe headache and sweating and there was elevation of the blood pressure (systolic: $170 \mathrm{mmHg}$; diastolic: $100 \mathrm{mmHg}$ ) which persisted throughout the stimulation, but when this was stopped the blood pressure soon returned to normal, and the headache and sweating disappeared. Then urine was collected by catheter, and spermatozoa were not found in the urinary sediment. On 31 July 1989 electroejaculation was repeated, with similar severe headache and a marked rise in blood pressure. After the two attempts the patient did not wish to try again; but he asked about some other method, and we advised him to try a papaverine hydrochloride intracavernous injection in August 1990. Since he could not use his hands, his cohabitant nurse did the injection. Each dose of papaverine hydrochloride was set for $20 \mathrm{mg}$ and 5 minutes after the injection full erection occured lasting for about 35 to 40 minutes. Subsequently the patient tried the injection three to four times a month and had sexual intercourse with his cohabitant. About February 1991 he began to notice that ejaculation was accompanied by spasms of the lower limbs, and was preceded by headache and perspiration about 30 minutes 
after the injection when the erection was about to be lost; by this time the cumulative dose of papaverine hydrochloride amounted to $440 \mathrm{mg}$. According to the patient, orgasm did not occur with ejaculation. Unfortunately the number of sperms and their mobility were not examined. In August 1991 his partner was found to be in her third month of pregnancy. The couple married in November 1991 and on 16 March 1992 she delivered transvaginally a healthy female child weighing $3330 \mathrm{~g}$.

\section{Discussion}

We have used the intracavernous self injection of vasoactive drugs in 68 patients with erectile dysfunction. Forty-two of them had a spinal cord injury, and of these patients only four $(10 \%)$ had penile erection with ejaculation. Fourteen patients $(21 \%)$ of the 68 patients, including eight patients with organic or combined impotence and six patients with functional impotence had varying degrees of ejaculation following self injection therapy. The mechanism of erection and ejaculation remains unknown in many respects. But it is known that erection is caused mainly through the pudendal nerve (somatic nerve) and pelvic nerve (parasympathetic nerve) while the hypogastric nerve (sympathetic nerve) plays a predominant role in ejaculation. Vasoactive drugs such as papaverine hydrochloride and prostaglandin $E_{1}$ are known to cause erection by acting on the corpus cavernosum, in the main by loosening its smooth muscle. ${ }^{1}$ It is difficult to directly link the intracavernous injection of a vasoactive drug to ejaculation. The mechanism of ejaculation which occurred in our patient is difficult to explain.

In the past, subarachnoid injection of neostigmine was widely used in the treatment of ejaculatory disturbance resulting from a spinal cord injury. In our department this procedure was used in a patient with a spinal cord injury successfully, leading to pregnancy and childhood. ${ }^{3}$ But the therapy was found to have many problems concerning safety and its use has now become infrequent. But electroejaculation, ${ }^{4,5}$ with electric stimulation, has replaced it and has gained the status of a mainstream therapy owing to its higher level of safety. The treatment of ejaculation disturbance is carried out entirely separately from the treatment of erectile dysfunction. Electroejaculation must be performed in the hospital setting, and artificial insemination of the wife with the semen obtained is required. This certainly creates a burden for patients and requires much effort on the part of the medical profession. Regarding the patient under consideration, we tried electroejaculation twice, but had to give up further trials because of the development of serious side effects. We thus changed the treatment for erectile dysfunction to intracavernous injection therapy and ejaculation occurred, leading to a successful pregnancy and childbirth by normal coitus. The lack of documented cases of partner pregnancy and childbirth resulting from the intracavernous injection of a vasoactive drug has prompted us to present this case.

\section{References}

1 Virag R, Shoukry K, Floresco J, Nollet F, Greco E (1991) Intracavernous self-injection of vasoactive drugs in the treatment of impotence: 8-year experience with 615 cases. J Urol 145: 287-293.

2 Otani T, Ohmura M, Itoh Y, Narushima M (1989) Administration of electrostimulation in the treatment of ejaculatory disturbance. JJTOM 37: 26-30.

3 Otani T, Kondo A, Takita T (1985) A paraplegic fathering a child after an intrathecal injection of neostigmine: Case report. Paraplegia 23: 32-37.

4 Brindley GS (1984) The fertility of men with spinal injuries. Paraplegia 22: 337-348.

5 Halstead LS, VerVoort S, Seager SW (1986) Rectal probe electrostimulation in the treatment of anejaculatory spinal cord injured men. Paraplegia 25: 120-129. 